\title{
Flexibility in Human Cognitive Behavior toward Nature*
}

\author{
Seizo OHE \\ Nihon University, Tokyo
}

Nearly forty years ago, I was a student of philosophy at Heidelberg in Germany and heard much about the so-called "epistemological subject". It is, as Heinrich Rickert himself once formulated, "just like a geometrical point in an epistemological space". At first, I was quite amazed at this superhumanly abstract image of the knowing man, but later on I found out that this superhumanly idealized image of the human intellectual ego is deeply rooted in the Western tradition of philosophical thinking, in modern times classically represented by the Kantian "transcendental ego". If such is the only legitimate concept of the cognitive subject, there can correspondingly be one and only one right way of our cognition of nature, and it was for Kant the Newtonian physics. On this point, however, we are today forced to think differently. In fact, man's cognitive behavior toward nature has always been more flexible, but an established culture often has its own rigid frame of mind, which needs to be broken from time to time. Now it may be time to re-examine man's cognitive behavior toward nature as a whole.

For some twenty years, I have been following this line of thought in my own way. In this paper, I will try to reach a certain, conclusive point of view, briefly recapitulating some of the most important results hitherto attained.

\section{I}

Let us first consider the world of our sense perception. The world in which we live everyday is essentially a perceptual world which cannot be much different from that of our remote ancestors. The mathematical science of nature has changed the interpretation of our perceptual world, but not this perceptual world as such. Modern civilization has transformed the world we perceive, but not the nature of our sense perception itself. There is, however, a strong traditional bias against this perceptual world. Kant, for instance, thought that the world of our sense perception is a world of sheer disorder and has no proper cognitive value. Is our perceptual world really such a chaos devoid of any orderly structure of its own?

* Read in another version at the 3rd ICLMPS, Amsterdam 1967 (Abstracts of Papers, P. 93). 
About ten years ago, I made an examination of our sense perception, and have specifically shown, by means of the mathematical concept of group, how, for exmaple, the world of our colored vision is based upon a closed and properly ordered structure of its own, essentially different from the physical world of visible light. In summary, when we focus our attention primarily on hues, disregarding saturation and brightness, the system of our colored vision forms a closed system very similar to that of a mathematical group, if we interpret color-mixing as a binary operation, neutral white as the identity element, and complimentary colors as the inverse elements. This is the so-called "color circle" of psychology. In contrast to this circle of sensory colors, the physical system of visible light forms only a small section of the whole spectrum of electromagnetic waves, the so-called "colorband", and it is rather natural that the perceptual element which closes the color circle, purple, does not exist among the spectral colors of this physical color band.

Let me give you one more example. According to the acoustic experiment of Wolfgang Köhler around 1910, in the frequency series of pure physical tones, characteristic U-, O-, A-, ä-, E-, I-vowel qualities are statistically confirmable in tones with a spacing of approximately one octave apart. Again, remarkably, in this series of pure physical tones neither ä- nor ü-qualities were found, the two vowel qualities which close the vowel system to a characteristic double triangle as is well-known in modern phonetics. An analogy to the former example of colors is at hand. And this system of spoken vowels can again be interpreted as forming a group-like structure, in this case, the smaller triangle AEO forming a subgroup with the so-called neutral vowel $\partial$ in the middle as the identity element, and by assuming a corresponding absolute neutral element, the so-called "white noise", in the middle of the larger triangle AIU, we can see the group-like structure of the whole vowel system in principle established.

Since colors and vowels are actually the perceptual substance of our external world in the sense that we normally see everything in color and hear every sound, more or less, vocalized, the above two examples might suffice to convince you that the world of our sense perception is not a chaos but a cosmos per se, and as such must have its own cognitive value.

II

Among the criticisms raised against the above arguments concerning the group-like structure of the color circle and of the vowel triangle, the most serious one was the following. - What would be the result, if we mix two just distinguishable color- or vowel-elements, say $A$ and $B$ ? Then the mixture we get thereby will be neither distinguishable from $A$ nor from $B$, and so the analogy with the mathematical group concept breaks down. Very true indeed! Precisely that is why I don't speak of a group, but of a group-like structure. In it I do not see the 
mathematical group concept itself, but only its psycho-physiological root, so to speak. No wonder if we cannot find here the perfect accuracy we have in fully developed mathematical thinking.

What is then the logico-epistemological principle which specifically characterizes mathematical thinking in general ? Let us take a look at one of the most popular textbooks of mathematical logic of today, Hilbert-Ackermann's. Written in terms of negation - and disjunction $\mathrm{v}$, the four axioms of its propositional logic are:
(1) $(\overline{x \vee x}) \vee x$,
(2) $\bar{x} \mathrm{\vee}(x \vee y)$,
(3) $(\overline{x \vee y}) \vee(y \vee x)$,
(4) $(\overline{\bar{x} \mathrm{\vee} y}) \vee[\overline{(z \vee} x) \vee(z \mathrm{\vee} y)]$.

They can be transformed by the usual rules of logical calculus into the following three disjunctive normal forms, whereby axioms (2) and (3) are turned into one and the same form (ii):

(i) $x \mathrm{v} \bar{x}$,

(ii) $x y \mathrm{\vee} \bar{x} y \mathrm{\vee} x \bar{y} \mathrm{v} \bar{x} \bar{y}$,

(iii) $x y z \vee \bar{x} y z \mathrm{v} x \bar{y} z \mathrm{v} x y \bar{z} \mathrm{v} \bar{x} \bar{y} z \mathrm{v} x \bar{y} \bar{z} \mathrm{v} \bar{x} y \bar{z} \mathrm{v} \bar{x} \bar{y} \bar{z}$.

These, in turn, can be represented by the following three corresponding square diagrams:
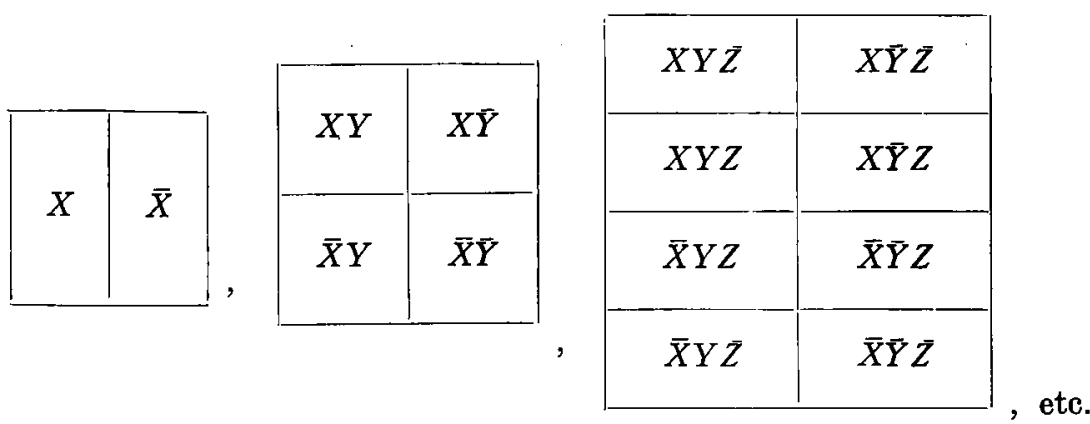

When we interpret $\mathrm{X}, \mathrm{Y}, \mathrm{Z}$ as meaning different predicates, we see here that the world is going to be divided into $2,4,8, \ldots$ parts by $1,2,3, \ldots$ predicates respectively. When we further transform the above three disjunctive normal forms into the following three corresponding conjunctive normal forms, whereby form (i) remains the same : 
(i) $\quad x \vee \bar{x}$,

(ii) $\quad(x \vee \bar{x})(y \vee \bar{y})$,

(iii) $^{\prime}(x \vee \bar{x})(y \vee \bar{y})(z \vee \bar{z})$.

Then we see that the original four axioms are now reducible to the following one mathematical formula: ${ }_{i=1}^{n} X_{i} \vee \bar{X}_{i}$, which means nothing else than the $n$-time application of the logical principle of the excluded middle to the world of discourse.

From the standpoint of ordinary experience, there is something humanly very natural in this way of thinking crudely dichotomic of the classical logic. It is quite natural, therefore, that the whole scientific development in modern times, above all the classical Newtonian physics, was ultimately based upon this dichtomic principle. By the same token, however, it is also quite understandable that this logico-epistemological principle of clear-cut dichotomy proved to be inadequate for describing microphysical experience now open to science. On the other hand, we saw already that this strictly dichotomic framework of experience did not entirely fit our sense perception. We saw namely that the mixture of two just distinguishable perceptual elements, say $A$ and $B$, cannot yield any new element with both qualities of $\mathrm{A}$ and $\mathrm{B}$, and distinguishable from both $\mathrm{A}$ and $\mathrm{B}$, as it should be according to the principle of clear-cut dichotomy.

It is indeed remarkable that a similar logico-epistemological difficulty arises in two apparently so different fields as sense perception and microphysics. One of the most striking results of contemprory physics is that light rays which had firmly been believed to be waves, were found to have also some irrefutable evidence for being of particle nature, and electrons which have been no less firmly believed to be charged particles with a definite mass, show, on the contrary, some indubitable characteristics of wave nature. All in all, one thing may now be regarded as established: the world of our classical science of nature based upon the dichotomic principle of the exculded middle is delimited at both ends, as it were, at its lower end by the world of sense perception and at its upper end by the world of modern physics.

\section{III}

We are thus coming to divide the whole world of our external knowledge into three specific worlds, which structurally differ in their basic modes of experience from one another. Let us tentatively call them "the world of sense perception", "the world of classical physics", and "the world of modern physics" respectively, representing them, for covenience' sake, by $\mathrm{P}, \mathrm{C}$, and $\mathrm{M}$ in the following diagrams, and think about possible relationships among them: 
1.

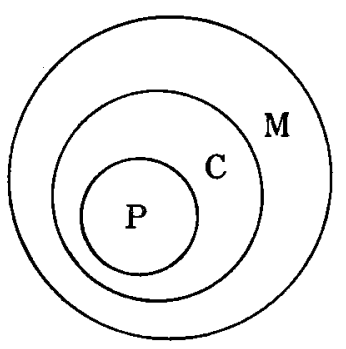

2.

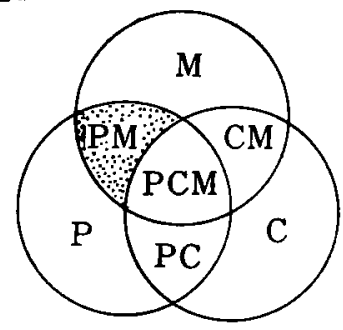

3.

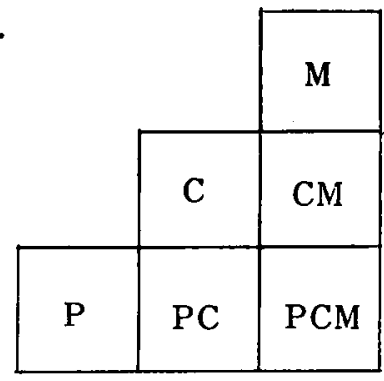

The first diagram faithfully reflects the whole process of the ever expanding science of nature from $\mathrm{P}$ to $\mathrm{C}$ and from $\mathrm{C}$ to $\mathrm{M}$. This is a particular relationship of one-sided inclusion, justifiable only from the theoretical viewpoint of physical science.

The second diagram just tries to correct this one-sidedness, and transforms the whole into a figure of three symmetrically overlapping circles. It certainly does justice to the relative independence of $\mathrm{P}, \mathrm{C}$, and $\mathrm{M}$, but if we consider the peculiar circumstance that any experimental contact between $\mathbf{P}$ and $\mathbf{M}$ so far is only possible through mediation by C, we have to strike out its PM-area, or better rearrange the whole into an entirely new figure like the third diagram, which does not contain any PM-area from the outset.

In conclusion, we now have, as a matter of fact, at least three kinds of cognitive behavior toward nature, each of which has its own raison d'être, its proper field of activity, and its appropriate epistemological interpretation:

1. The cognitive behavior of perceptual experience. Ordinarily we live in this world of sense perception. As long as we perceive a color as a color and not as an electromagnetic wave, our sensory perception has its own value, not only an aesthetic but also a cognitive value. Epistemologically, the most adequate standpoint hereto may be the so-called "naive realism".

2. The cognitive behavior of classical physics. As long as our ordinary experience with its deterministic frame of reference actually subsists, classical physics never loses its raison d'être, and may well deserve further systematic development on its own account. Its appropriate epistemology may be the socalled "critical realism".

3. The cognitive behavior of modern physics. Modern physics deals with phenomena far beyond our ordinary experience and requires accordingly an entirely new conceptual frame with its principle of indeterminancy. The "positivistic" or "phenomenalistic" epistemology might here be the most consequent.

All three are important for us. Their relative independence is no less im- 
portant than their basic interdependence. Such a multiplicity of cognitive behavior necessarily requires a corresponding flexibility of mind, a kind of intellectual mobility among these three possibilities. With due efforts, we can switch over, so to speak, from one behavior to another, as circumstances demand. The scientific investigation of nature need not be bound to any rigid standpoint in phiolsophical epistemology. Scientific phiolsophy has to do justice to the ever changing situation of human knowledge with that admirable flexibility which has always been one of the most distinctive characteristics of human intelligenec.

(Received Jan. 16, 1968)

N.B. Related papers by the same author so far published in Western languages:

1. La structure mathématique des sensations, Proc. XI. Interm. Congr. Philos., Brussels, 1953.

2. Die mehrfache Struktur der Naturerkenntnis, Proc. II. Intern. Congr. of the U.I.P.S', Zürich, 1954.

3. Le problème de la réalité objective et la structure multiple de notre connaissance extérieure, Rev. Philsophique, LXXIX-7/9, Paris, 1954.

4. Application of mathematical group concept to human perceptual systems, Ann. of the Japan Association for Philosophy of Science, 1-2, Tokyo, 1957.

5. Land's experiments in color vision and the mathematical structure of our sense perception, Ann. of the J.A.P.S., II-2, Tokyo, 1962.

6. La méthode axiomatique et la structure multiple de notre connaissance extérieure, La Méthode Axiomatique dans les Mécaniques, Classiques et Nouvelles (Les Grands Problèmes des Sciences 13), Gauthier-Villars, Paris, 1963.

7. The multiple structure of our external knowledge, The Philosophical Studies of Japan, V, Unesco-Tokyo, 1964. 